\title{
ELZAKI TRANSFORM HOMOTOPY PERTURBATION METHOD FOR SOLVING POROUS MEDIUM EQUATION
}

\author{
Prem Kiran G. Bhadane', V. H. Pradhan ${ }^{2}$ \\ ${ }^{1}$ Assistant Professor, Department of Applied Science, RCPIT, Maharashtra, India, \\ omprem07@gmail.com \\ ${ }^{2}$ Associate Professor, Department of Applied Mathematics and Humanities, SVNIT, Gujarat, India \\ pradhan65@yahoo.com
}

\begin{abstract}
In this paper, we apply a new method called ELzaki transform homotopy perturbation method (ETHPM) to solve porous medium equation. This method is a combination of the new integral transform "ELzaki transform" and the homotopy perturbation method. The nonlinear term can be easily handled by homotopy perturbation method. The porous medium equations have importance in engineering and sciences and constitute a good model for many systems in various fields. Some cases of the porous medium equation are solved as examples to illustrate ability and reliability of mixture of ELzaki transform and homotopy perturbation method. The results reveal that the combination of ELzaki transform and homotopy perturbation method is quite capable, practically well appropriate for use in such problems and can be applied to other nonlinear problems. This method is seen as a better alternative method to some existing techniques for such realistic problems.
\end{abstract}

Key words: ELzaki transform, Homotopy perturbation method, non linear partial differential equation, and porous medium equation

\section{INTRODUCTION}

Many of the physical phenomena and processes in various fields of engineering and science are governed by partial differential equations. The nonlinear heat equation describing various physical phenomena called the porous medium equation. The porous medium equation [3] is

$\frac{\partial \mathrm{u}}{\partial \mathrm{t}}=\frac{\partial}{\partial \mathrm{x}}\left(\mathrm{u}^{\mathrm{m}} \frac{\partial \mathrm{u}}{\partial \mathrm{x}}\right)$

where $\mathrm{m}$ is a rational number. There are number of physical applications where this simple model appears in a natural way, mainly to describe processes involving fluid flow, heat transfer or diffusion. May be the best known of them is the description of the flow of an isentropic gas through a porous medium, modeled independently by Leibenzon and Muskat around 1930. Also application is found in the study of groundwater infiltration by Boussisnesq in 1903. Another important application refers to heat radiation in plasmas, developed by Zel'dovich and coworkers around 1950. All of these reasons support the interest of its study both for the mathematician and the scientist.

In recent years, many research workers have paid attention to find the solution of nonlinear differential equations by using various methods. Among these are the Adomian decomposition method [Hashim, Noorani, Ahmed. Bakar, Ismail and Zakaria, (2006)], the tanh method, the homotopy perturbation method [ Sweilam, Khader (2009), Sharma and Giriraj Methi (2011), Jafari, Aminataei (2010), (2011) ], the differential transform method [(2008)], homotopy perturbation transform method and the variational iteration method. Various ways have been proposed recently to deal with these nonlinearities; one of these combinations is ELzaki transform and homotopy perturbation method. ELzaki transform is a useful technique for solving linear differential equations but this transform is totally incapable of handling nonlinear equations [4] because of the difficulties that are caused by the nonlinear terms. This paper is using homotopy perturbation method to decompose the nonlinear term, so that the solution can be obtained by iteration procedure. This means that we can use both ELzaki transform and homotopy perturbation methods to solve many nonlinear problems. The main aim of this paper is to consider the effectiveness of the Elzaki transform homotopy perturbation method in solving nonlinear porous medium equations. This method provides the solution in a rapid convergent series which may leads the solution in a closed form. The fact that the proposed technique solves nonlinear problems without using so-called Adomian's polynomials is a clear advantage of this algorithm over the decomposition method.

\section{ELZAKI TRANSFORM HOMOTOPY PERTURBATION METHOD [4]}

Consider a general nonlinear non-homogenous partial differential equation with initial conditions of the form: $\operatorname{Du}(\mathrm{x}, \mathrm{t})+\operatorname{Ru}(\mathrm{x}, \mathrm{t})+\mathrm{Nu}(\mathrm{x}, \mathrm{t})=\mathrm{g}(\mathrm{x}, \mathrm{t})$ 


$$
\mathrm{u}(\mathrm{x}, 0)=\mathrm{h}(\mathrm{x}) \quad, \quad \mathrm{u}_{\mathrm{t}}(\mathrm{x}, 0)=\mathrm{f}(\mathrm{x})
$$

where $\mathrm{D}$ is linear differential operator of order two, $\mathrm{R}$ is linear differential operator of less order than $\mathrm{D}, \mathrm{N}$ is the general nonlinear differential operator and $g(x, t)$ is the source term.

Taking ELzaki transform on both sides of equation (2), to get:

$$
E[\operatorname{Du}(x, t)]+E[R u(x, t)]+E[N u(x, t)]=E[g(x, t)]
$$

Using the differentiation property of ELzaki transform and above initial conditions, we have:

$$
\begin{array}{r}
E[u(x, t)]=v^{2} E[g(x, t)]+v^{2} h(x)+v^{3} f(x) \\
-v^{2} E[R u(x, t)+N u(x, t)]
\end{array}
$$

Applying the inverse ELzaki transform on both sides of equation (4), to find:

$\mathrm{u}(\mathrm{x}, \mathrm{t})=\mathrm{G}(\mathrm{x}, \mathrm{t})-\mathrm{E}^{-1}\left\{\mathrm{v}^{2} \mathrm{E}[\mathrm{Ru}(\mathrm{x}, \mathrm{t})+\mathrm{Nu}(\mathrm{x}, \mathrm{t})]\right\}$

where $G(x, t)$ represents the term arising from the source term and the prescribed initial condition.

Now, we apply the homotopy perturbation method,

$$
\mathrm{u}(\mathrm{x}, \mathrm{t})=\sum_{\mathrm{n}=0}^{\infty} \mathrm{p}^{\mathrm{n}} \mathrm{u}_{\mathrm{n}}(\mathrm{x}, \mathrm{t})
$$

and the nonlinear term can be decomposed as

$\mathrm{N}[\mathrm{u}(\mathrm{x}, \mathrm{t})]=\sum_{\mathrm{n}=0}^{\infty} \mathrm{p}^{\mathrm{n}} \mathrm{H}_{\mathrm{n}}(\mathrm{u})$

where $\mathrm{H}_{\mathrm{n}}(\mathrm{u})$ are He's polynomial and given by

$$
\begin{gathered}
\mathrm{H}_{\mathrm{n}}\left(\mathrm{u}_{0}, \mathrm{u}_{1}, \ldots \ldots, \mathrm{u}_{\mathrm{n}}\right)=\frac{1}{\mathrm{n} !} \frac{\partial^{\mathrm{n}}}{\partial \mathrm{p}^{\mathrm{n}}}\left[\mathrm{N}\left(\sum_{\mathrm{i}=0}^{\infty} \mathrm{p}^{\mathrm{i}} \mathrm{u}_{\mathrm{i}}\right)\right]_{\mathrm{p}=0}, \\
\mathrm{n}=0,1,2, \ldots \ldots \ldots
\end{gathered}
$$

Substituting equations (6) and (7) in equation (5), we get:

$$
\begin{aligned}
& \sum_{n=0}^{\infty} p^{n} u_{n}(x, t)=G(x, t)- \\
& p\left\{E^{-1}\left[v^{2} E\left[R \sum_{n=0}^{\infty} p^{n} u_{n}(x, t)+\sum_{n=0}^{\infty} p^{n} H_{n}(u)\right]\right]\right\}
\end{aligned}
$$

This is the coupling of the ELzaki transform and the homotopy perturbation method. Comparing the coefficient of like powers of $\mathrm{p}$, the following approximations are obtained.

$\mathrm{p}^{0}: \mathrm{u}_{0}(\mathrm{x}, \mathrm{t})=\mathrm{G}(\mathrm{x}, \mathrm{t})$

$\mathrm{p}^{1}: \mathrm{u}_{1}(\mathrm{x}, \mathrm{t})=-\mathrm{E}^{-1}\left\{\mathrm{v}^{2} \mathrm{E}\left[\mathrm{Ru}_{0}(\mathrm{x}, \mathrm{t})+\mathrm{H}_{0}(\mathrm{u})\right]\right\}$

$\mathrm{p}^{2}: \mathrm{u}_{2}(\mathrm{x}, \mathrm{t})=-\mathrm{E}^{-1}\left\{\mathrm{v}^{2} \mathrm{E}\left[\mathrm{Ru}_{1}(\mathrm{x}, \mathrm{t})+\mathrm{H}_{1}(\mathrm{u})\right]\right\}$

$p^{3}: u_{3}(x, t)=-E^{-1}\left\{v^{2} E\left[R_{2}(x, t)+H_{2}(u)\right]\right\}$

Then the solution is

$\mathrm{u}(\mathrm{x}, \mathrm{t})=\lim _{\mathrm{p} \rightarrow 1} \mathrm{u}_{\mathrm{n}}(\mathrm{x}, \mathrm{t})$

$$
=\mathrm{u}_{0}(\mathrm{x}, \mathrm{t})+\mathrm{u}_{1}(\mathrm{x}, \mathrm{t})+\mathrm{u}_{2}(\mathrm{x}, \mathrm{t})+\ldots
$$

\section{APPLICATIONS}

Now, we consider in this section the effectiveness of the ELzaki transform homotopy-perturbation method to obtain the exact and approximate analytical solution of the porous medium equations.

Example 3.1 Let us take $m=-1$ in equation (1), we get

$$
\frac{\partial \mathrm{u}}{\partial \mathrm{t}}=\frac{\partial}{\partial \mathrm{x}}\left(\left(\mathrm{u}^{-1}\right) \frac{\partial \mathrm{u}}{\partial \mathrm{x}}\right)
$$

with initial condition as $\mathrm{u}(\mathrm{x}, 0)=\frac{1}{\mathrm{x}}$.

Exact solution [1] of this equation is $u(x, t)=\left(c_{1} x-c_{1}^{2} t+\right.$ $\left.c_{2}\right)^{-1}$ with the values of arbitrary constants taken as $\mathrm{c}_{1}=1$ and $\mathrm{c}_{2}=0$ solution becomes $\mathrm{u}(\mathrm{x}, \mathrm{t})=\frac{1}{\mathrm{x}-\mathrm{t}}$.

We can find solution by applying ELzaki transform on both side of equation (11) subject to the initial condition

$$
E\left[\frac{\partial u}{\partial t}\right]=E\left[\left(u^{-1}\right) \frac{\partial^{2} u}{\partial x^{2}}-\left(u^{-2}\right)\left(\frac{\partial u}{\partial x}\right)^{2}\right]
$$

This can be written as

$$
\begin{aligned}
\frac{1}{\mathrm{v}} \mathrm{E}[\mathrm{u}(\mathrm{x}, \mathrm{t})]-\mathrm{vu} & (\mathrm{x}, 0) \\
& =\mathrm{E}\left[\left(\mathrm{u}^{-1}\right) \frac{\partial^{2} \mathrm{u}}{\partial \mathrm{x}^{2}}-\left(\mathrm{u}^{-2}\right)\left(\frac{\partial \mathrm{u}}{\partial \mathrm{x}}\right)^{2}\right]
\end{aligned}
$$

On applying the above specified initial condition, we get

$$
\mathrm{E}[\mathrm{u}(\mathrm{x}, \mathrm{t})]=\mathrm{v}^{2} \frac{1}{\mathrm{x}}+\mathrm{vE}\left[\left(\mathrm{u}^{-1}\right) \frac{\partial^{2} \mathrm{u}}{\partial \mathrm{x}^{2}}-\left(\mathrm{u}^{-2}\right)\left(\frac{\partial \mathrm{u}}{\partial \mathrm{x}}\right)^{2}\right]
$$

Taking inverse ELzaki transform on both sides of Eq. (14), we get

$$
\mathrm{u}(\mathrm{x}, \mathrm{t})=\frac{1}{\mathrm{x}}+\mathrm{E}^{-1}\left\{\mathrm{vE}\left[\left(\mathrm{u}^{-1}\right) \frac{\partial^{2} \mathrm{u}}{\partial \mathrm{x}^{2}}-\left(\mathrm{u}^{-2}\right)\left(\frac{\partial \mathrm{u}}{\partial \mathrm{x}}\right)^{2}\right]\right\}
$$

Now we apply the homotopy perturbation method,

$\mathrm{u}(\mathrm{x}, \mathrm{t})=\sum_{\mathrm{n}=0}^{\infty} \mathrm{p}^{\mathrm{n}} \mathrm{u}_{\mathrm{n}}(\mathrm{x}, \mathrm{t})$

and the nonlinear term can be decomposed as

$\mathrm{N}[\mathrm{u}(\mathrm{x}, \mathrm{t})]=\sum_{\mathrm{n}=0}^{\infty} \mathrm{p}^{\mathrm{n}} \mathrm{H}_{\mathrm{n}}(\mathrm{u})$

Using Eqs. (16)- (17) into Eq. (15), we get

$$
\sum_{n=0}^{\infty} p^{n} u_{n}(x, t)=\frac{1}{x}+p E^{-1}\left\{v E\left[\sum_{n=0}^{\infty} p^{n} H_{n}(u)\right]\right\}
$$

where $\mathrm{H}_{\mathrm{n}}(\mathrm{u})$ are He's polynomials. The first two components of He's polynomials are given by

$$
\begin{aligned}
\mathrm{H}_{0}(\mathrm{u})=\left(\mathrm{u}_{0}^{-1}\right) \frac{\partial^{2} \mathrm{u}_{0}}{\partial \mathrm{x}^{2}}-\left(\mathrm{u}_{0}^{-2}\right)\left(\frac{\partial \mathrm{u}_{0}}{\partial \mathrm{x}}\right)^{2} \\
\mathrm{H}_{1}(\mathrm{u})=\left(\mathrm{u}_{0}^{-1}\right)\left(-\frac{\mathrm{u}_{1}}{\mathrm{u}_{0}} \frac{\partial^{2} \mathrm{u}_{0}}{\partial \mathrm{x}^{2}}+\frac{\partial^{2} \mathrm{u}_{1}}{\partial \mathrm{x}^{2}}\right) \\
-\left(\mathrm{u}_{0}^{-2}\right)\left(-2 \frac{\mathrm{u}_{1}}{\mathrm{u}_{0}}\left(\frac{\partial \mathrm{u}_{0}}{\partial \mathrm{x}}\right)^{2}+2 \frac{\partial \mathrm{u}_{0}}{\partial \mathrm{x}} \frac{\partial \mathrm{u}_{1}}{\partial \mathrm{x}}\right)
\end{aligned}
$$

Comparing the coefficient of various power of $p$ in (18), we get

$$
\begin{aligned}
& \mathrm{p}^{0}: \mathrm{u}_{0}(\mathrm{x}, \mathrm{t})=\frac{1}{\mathrm{x}}, \\
& \begin{array}{r}
\mathrm{p}^{1}: \mathrm{u}_{1}(\mathrm{x}, \mathrm{t})=\mathrm{E}^{-1}\left\{\mathrm{vE}\left[\mathrm{H}_{0}(\mathrm{u})\right]\right\} \\
=\mathrm{E}^{-1}\left\{\mathrm{vE}\left[\left(\mathrm{u}_{0}^{-1}\right) \frac{\partial^{2} \mathrm{u}_{0}}{\partial \mathrm{x}^{2}}-\left(\mathrm{u}_{0}^{-2}\right)\left(\frac{\partial \mathrm{u}_{0}}{\partial \mathrm{x}}\right)^{2}\right]\right\} \\
=\frac{\mathrm{t}}{\mathrm{x}^{2}},
\end{array} \\
& \mathrm{p}^{2}: \mathrm{u}_{2}(\mathrm{x}, \mathrm{t})=\mathrm{E}^{-1}\left\{\mathrm{vE}\left[\mathrm{H}_{1}(\mathrm{u})\right]\right\} \\
& =\mathrm{E}^{-1}\left\{\mathrm { vE } \left[\left(\mathrm{u}_{0}^{-1}\right)\left(-\frac{\mathrm{u}_{1}}{\mathrm{u}_{0}} \frac{\partial^{2} \mathrm{u}_{0}}{\partial \mathrm{x}^{2}}+\frac{\partial^{2} \mathrm{u}_{1}}{\partial \mathrm{x}^{2}}\right)^{2}\right.\right. \\
& \left.\left.\quad-\left(\mathrm{u}_{0}^{-2}\right)\left(-2 \frac{\mathrm{u}_{1}}{\mathrm{u}_{0}}\left(\frac{\partial \mathrm{u}_{0}}{\partial \mathrm{x}}\right)^{2}+2 \frac{\partial \mathrm{u}_{0}}{\partial \mathrm{x}} \frac{\partial \mathrm{u}_{1}}{\partial \mathrm{x}}\right)\right]\right\}
\end{aligned}
$$




$$
=\frac{t^{2}}{x^{3}}
$$

Proceeding in similar manner we can obtain further values, substituting above values in equation (10), we get solution in the form of a series

$$
u(x, t)=\frac{1}{x}+\frac{t}{x^{2}}+\frac{t^{2}}{x^{3}}+\frac{t^{3}}{x^{4}} \ldots \ldots=\frac{1}{x-t}
$$

This is the solution of (11) and which is exactly the exact solution given above.

Example 3.2 Let us take $m=1$ in equation (1), we get

$\frac{\partial \mathrm{u}}{\partial \mathrm{t}}=\frac{\partial}{\partial \mathrm{x}}\left(\mathrm{u} \frac{\partial \mathrm{u}}{\partial \mathrm{x}}\right)$

with initial condition as $\mathrm{u}(\mathrm{x}, 0)=\mathrm{x}$.

Exact solution [1] of this equation is $u(x, t)=x+$ $\left(c_{1}+c_{2}\right) t$ with the values of arbitrary constants taken as $\mathrm{c}_{1}=1$ and $\mathrm{c}_{2}=0$ solution becomes $\mathrm{u}(\mathrm{x}, \mathrm{t})=\mathrm{x}+\mathrm{t}$.

we can find solution by applying ELzaki transform on both side of equation (20)

$E\left[\frac{\partial u}{\partial t}\right]=E\left[u \frac{\partial^{2} u}{\partial x^{2}}+\left(\frac{\partial u}{\partial x}\right)^{2}\right]$

This can be written as

$\frac{1}{\mathrm{v}} \mathrm{E}[\mathrm{u}(\mathrm{x}, \mathrm{t})]-\mathrm{vu}(\mathrm{x}, 0)=\mathrm{E}\left[\mathrm{u} \frac{\partial^{2} \mathrm{u}}{\partial \mathrm{x}^{2}}+\left(\frac{\partial \mathrm{u}}{\partial \mathrm{x}}\right)^{2}\right]$

On applying the above specified initial condition, we get

$E[u(x, t)]=v^{2} x+v E\left[u \frac{\partial^{2} u}{\partial x^{2}}\left(\frac{\partial u}{\partial x}\right)^{2}\right]$

Taking inverse ELzaki transform on both sides of Eq. (23), we get

$$
u(x, t)=x+E^{-1}\left\{v E\left[u \frac{\partial^{2} u}{\partial x^{2}}+\left(\frac{\partial u}{\partial x}\right)^{2}\right]\right\}
$$

Now we apply the homotopy perturbation method,

$$
\mathrm{u}(\mathrm{x}, \mathrm{t})=\sum_{\mathrm{n}=0}^{\infty} \mathrm{p}^{\mathrm{n}} \mathrm{u}_{\mathrm{n}}(\mathrm{x}, \mathrm{t})
$$

and the nonlinear term can be decomposed as

$$
\mathrm{N}[\mathrm{u}(\mathrm{x}, \mathrm{t})]=\sum_{\mathrm{n}=0}^{\infty} \mathrm{p}^{\mathrm{n}} \mathrm{H}_{\mathrm{n}}(\mathrm{u})
$$

Invoking Eqs. (25)- (26) into Eq. (24), we get

$\sum_{n=0}^{\infty} p^{n} u_{n}(x, t)=x+p E^{-1}\left\{v E\left[\sum_{n=0}^{\infty} p^{n} H_{n}(u)\right]\right\}$

where $\mathrm{H}_{\mathrm{n}}(\mathrm{u})$ are He's polynomials. The first two components of He's polynomials are given by

$$
\begin{aligned}
& \mathrm{H}_{0}(\mathrm{u})=\mathrm{u}_{0} \frac{\partial^{2} \mathrm{u}_{0}}{\partial \mathrm{x}^{2}}+\left(\frac{\partial \mathrm{u}_{0}}{\partial \mathrm{x}}\right)^{2}, \\
& \mathrm{H}_{1}(\mathrm{u})=\left(\mathrm{u}_{1} \frac{\partial^{2} \mathrm{u}_{0}}{\partial \mathrm{x}^{2}}+\mathrm{u}_{0} \frac{\partial^{2} \mathrm{u}_{1}}{\partial \mathrm{x}^{2}}\right)+2 \frac{\partial \mathrm{u}_{0}}{\partial \mathrm{x}} \frac{\partial \mathrm{u}_{1}}{\partial \mathrm{x}},
\end{aligned}
$$

Comparing the coefficient of various power of $p$ in (27), we get

$$
\begin{aligned}
& \mathrm{p}^{0}: \mathrm{u}_{0}(\mathrm{x}, \mathrm{t})=\mathrm{x}, \\
& \mathrm{p}^{1}: \mathrm{u}_{1}(\mathrm{x}, \mathrm{t})=\mathrm{E}^{-1}\left\{\mathrm{vE}\left[\mathrm{H}_{0}(\mathrm{u})\right]\right\} \\
&=\mathrm{E}^{-1}\left\{\mathrm{vE}\left[\mathrm{u}_{0} \frac{\partial^{2} \mathrm{u}_{0}}{\partial \mathrm{x}^{2}}+\left(\frac{\partial \mathrm{u}_{0}}{\partial \mathrm{x}}\right)^{2}\right]\right\}=\mathrm{t},
\end{aligned}
$$$$
\mathrm{p}^{2}: \mathrm{u}_{2}(\mathrm{x}, \mathrm{t})=\mathrm{E}^{-1}\left\{\mathrm{vE}\left[\mathrm{H}_{1}(\mathrm{u})\right]\right\}
$$

$$
\begin{aligned}
& =E^{-1}\left\{v E\left[u_{1} \frac{\partial^{2} u_{0}}{\partial x^{2}}+u_{0} \frac{\partial^{2} u_{1}}{\partial x^{2}}+2 \frac{\partial u_{0}}{\partial x} \frac{\partial u_{1}}{\partial x}\right]\right\} \\
& =0,
\end{aligned}
$$

$\mathrm{p}^{3}: \mathrm{u}_{3}(\mathrm{x}, \mathrm{t})=0, \mathrm{p}^{4}: \mathrm{u}_{4}(\mathrm{x}, \mathrm{t})=0$,

and so on we will found that $u_{n}(x, t)=0$ for $n \geq 2$.

Substituting above values in equation (10) we get solution in the form of a series

$\mathrm{u}(\mathrm{x}, \mathrm{t})=\mathrm{x}+\mathrm{t}+0+0 \ldots=\mathrm{x}+\mathrm{t}$

This is the solution of (20) and which is exactly the exact solution given above.

Example 3.3 Let us take $m=-4 / 3$ in equation (1), we get

$\frac{\partial \mathrm{u}}{\partial \mathrm{t}}=\frac{\partial}{\partial \mathrm{x}}\left((\mathrm{u})^{-4 / 3} \frac{\partial \mathrm{u}}{\partial \mathrm{x}}\right)$

With initial condition as $\mathrm{u}(\mathrm{x}, 0)=(2 \mathrm{x})^{-3 / 4}$.

Using afore said method, we have

$\mathrm{u}(\mathrm{x}, \mathrm{t})=(2 \mathrm{x})^{-3 / 4}$

$+\mathrm{E}^{-1}\left\{\mathrm{v} \mathrm{E}\left[(\mathrm{u})^{-4 / 3} \frac{\partial^{2} \mathrm{u}}{\partial \mathrm{x}^{2}}-\frac{4}{3}(\mathrm{u})^{-7 / 3}\left(\frac{\partial \mathrm{u}}{\partial \mathrm{x}}\right)^{2}\right]\right\}$

Now we apply the homotopy perturbation method,

$\mathrm{u}(\mathrm{x}, \mathrm{t})=\sum_{\mathrm{n}=0}^{\infty} \mathrm{p}^{\mathrm{n}} \mathrm{u}_{\mathrm{n}}(\mathrm{x}, \mathrm{t})$

and the nonlinear term can be decomposed as

$\mathrm{N}[\mathrm{u}(\mathrm{x}, \mathrm{t})]=\sum_{\mathrm{n}=0}^{\infty} \mathrm{p}^{\mathrm{n}} \mathrm{H}_{\mathrm{n}}(\mathrm{u})$

Invoking Eqs. (31)- (32) into Eq. (30), we get

$$
\begin{aligned}
& \sum_{n=0}^{\infty} p^{n} u_{n}(x, t) \\
& =(2 x)^{-3 / 4}+p^{-1}\left\{v E\left[\sum_{n=0}^{\infty} p^{n} H_{n}(u)\right]\right\}
\end{aligned}
$$

where $\mathrm{H}_{\mathrm{n}}(\mathrm{u})$ are He's polynomials. The first two components of He's polynomials are given by

$$
\begin{aligned}
\mathrm{H}_{0}(\mathrm{u})= & \left(\mathrm{u}_{0}\right)^{-4 / 3} \frac{\partial^{2} \mathrm{u}_{0}}{\partial \mathrm{x}^{2}}-\frac{4}{3}\left(\mathrm{u}_{0}\right)^{-7 / 3}\left(\frac{\partial \mathrm{u}_{0}}{\partial \mathrm{x}}\right)^{2}, \\
\mathrm{H}_{1}(\mathrm{u})= & \left(\mathrm{u}_{0}\right)^{-4 / 3}\left(\frac{\partial^{2} \mathrm{u}_{1}}{\partial \mathrm{x}^{2}}-\left(\frac{4}{3}\right) \frac{\partial^{2} \mathrm{u}_{0}}{\partial \mathrm{x}^{2}}\left(\frac{\mathrm{u}_{1}}{\mathrm{u}_{0}}\right)\right) \\
& -\frac{4}{3}\left(\mathrm{u}_{0}\right)^{-7 / 3}\left(2\left(\frac{\partial \mathrm{u}_{0}}{\partial \mathrm{x}}\right)\left(\frac{\partial \mathrm{u}_{1}}{\partial \mathrm{x}}\right)-\frac{7}{3}\left(\frac{\partial \mathrm{u}_{0}}{\partial \mathrm{x}}\right)^{2}\left(\frac{\mathrm{u}_{1}}{\mathrm{u}_{0}}\right)\right),
\end{aligned}
$$

Comparing the coefficient of various power of $p$ in (33), we get $\mathrm{p}^{0}: \mathrm{u}_{0}(\mathrm{x}, \mathrm{t})=(2 \mathrm{x})^{-3 / 4}$,

$\mathrm{p}^{1}: \mathrm{u}_{1}(\mathrm{x}, \mathrm{t})=\mathrm{E}^{-1}\left\{\mathrm{vE}\left[\mathrm{H}_{0}(\mathrm{u})\right]\right\}$

$=\mathrm{E}^{-1}\left\{\mathrm{vE}\left[\left(\mathrm{u}_{0}\right)^{-4 / 3} \frac{\partial^{2} \mathrm{u}_{0}}{\partial \mathrm{x}^{2}}-\frac{4}{3}\left(\mathrm{u}_{0}\right)^{-7 / 3}\left(\frac{\partial \mathrm{u}_{0}}{\partial \mathrm{x}}\right)^{2}\right]\right\}$

$=9 \times 2^{-15 / 4} \times x^{-7 / 4} \times t$,

$\mathrm{p}^{2}: \mathrm{u}_{2}(\mathrm{x}, \mathrm{t})=\mathrm{E}^{-1}\left\{\mathrm{vE}\left[\mathrm{H}_{1}(\mathrm{u})\right]\right\}$ 
$=\mathrm{E}^{-1}\left\{\begin{array}{c}\mathrm{vE}\left[\left(\mathrm{u}_{0}\right)^{-4 / 3}\left(\frac{\partial^{2} \mathrm{u}_{1}}{\partial \mathrm{x}^{2}}-\left(\frac{4}{3}\right) \frac{\partial^{2} \mathrm{u}_{0}}{\partial \mathrm{x}^{2}}\left(\frac{\mathrm{u}_{1}}{\mathrm{u}_{0}}\right)\right)\right] \\ -\mathrm{vE}\left[\frac{4}{3} \mathrm{u}_{0}^{-\frac{7}{3}}\left(2 \frac{\partial \mathrm{u}_{0}}{\partial \mathrm{x}} \frac{\partial \mathrm{u}_{1}}{\partial \mathrm{x}}-\frac{7}{3}\left(\frac{\partial \mathrm{u}_{0}}{\partial \mathrm{x}}\right)^{2}\left(\frac{\mathrm{u}_{1}}{\mathrm{u}_{0}}\right)\right)\right]\end{array}\right\}$

$=189 \times 2^{-31 / 4} \times \mathrm{x}^{-11 / 4} \times \mathrm{t}^{2}$,

On substituting these terms in equation (10), we obtained the solution

$$
\begin{gathered}
u(x, t)=(2 x)^{-3 / 4}+9 \times 2^{-15 / 4} \times x^{-7 / 4} \times t+189 \\
\times 2^{-31 / 4} \times x^{-11 / 4} \times t^{2}+\cdots
\end{gathered}
$$

This result can be verified through substitution.

\section{CONCLUSION}

The main goal of this paper is to show the applicability of the mixture of new integral transform "ELzaki transform" with the homotopy perturbation method to construct an analytical solution for porous medium equation. This combination of two methods successfully worked to give very reliable and exact solutions to the equation. This method provides an analytical approximation in a rapidly convergent sequence with in exclusive manner computed terms. Its rapid convergence shows that the method is trustworthy and introduces a significant improvement in solving nonlinear partial differential equations over existing methods.

\section{ACKNOWLEDGEMENT}

I am deeply grateful to the management of Shirpur Education Society, Shirpur (Maharashtra) without whose support my research work would not have been possible. I would also like to extend my gratitude to the Prin. Dr. J. B. Patil and Mr. S. P. Shukla, Head of Department of Applied Science, RCPIT for helping and inspiring me for the research work.

\section{REFERENCES}

[1] A.D. Polyanin, V.F. Zaitsev (2004), Handbook of Nonlinear Partial Differential Equations, Chapman and Hall/CRC Press, Boca Raton,.2004.

[2] Mishra D, Pradhan V. H., Mehta M. N. (2012), Solution of Porous Medium Equation by Homotopy Perturbation Transform Method, International Journal of Engineering Research and Applications, Vol.2 Issue 3, pp2041-2046.

[3] Juan Luis Vazquez (2007), The Porous Medium Equation Mathematical Theory, Oxford Science Publication, Clarenden Press, pp1-28.

[4] Tarig M. Elzaki and Eman M. A. Hilal (2012), Homotopy Perturbation and ELzaki Transform for solving Nonlinear Partial Differential equations, Mathematical Theory and Modeling, Vol.2,No.3, pp33-42.

[5] Tarig M. Elzaki and Salih M. Elzaki (2011), Applications of New Transform "ELzaki
Transform" to Partial Differential Equations, Global Journal of Pure and Applied Mathematics, Vol.7, No.1,pp65-70.

[6] Tarig M. Elzaki (2011), The New Integral Transform "ELzaki Transform", Global Journal of Pure and Applied Mathematics, Vol.7, No.1,pp5764.

[7] Tarig M. Elzaki, Salih M. Elzaki and Elsayed A. Elnour (2012), On the New Integral Transform "ELzaki Transform" Fundamental Properties Investigations and Applications, Global Journal of Mathematical Sciences: Theory and Practical, Vol.4, No.1, pp1-13. 